\title{
Acerca del fundamento de la necesidad de las leyes empíricas de la ciencia en un sentido impropio en Kant. Una crítica de Michael Bennett McNulty
}

\author{
On the Ground of the Necessity of Empirical Laws of Science in an \\ Improper Sense in Kant. A Critique of Michael Bennett McNulty
}

Martin Arias Albisu*

\begin{abstract}
Resumen: El objetivo de este trabajo es criticar un artículo de Michael B. McNulty publicado en 2015, a saber, "Rehabilitating the Regulative Use of Reason: Kant on Empirical and Chemical Laws". En este artículo, McNulty examina la concepción kantiana de la necesidad de las leyes empíricas pertenecientes a lo que, en los Primeros principios metafísicos de la ciencia de la naturaleza, se denomina ciencia en un sentido "impropio". McNulty considera la única ciencia en sentido impropio mencionada por Kant, a saber, la química. En su artículo, McNulty presenta una interpretación original e interesante de esa concepción. Según esta interpretación, ciertas ideas de la razón, que el autor llama "elementos", son el fundamento de la necesidad de las leyes químicas. Haremos tres críticas a esta interpretación. 1) La tesis de McNulty según la cual desde la perspectiva de la razón las leyes químicas son estrictamente necesarias es cognitivamente irrelevante para nosotros. 2) McNulty no menciona textos de Kant que muestren claramente que su interpretación del fundamento de la necesidad de las leyes químicas es correcta. 3) Nuestra interpretación de la necesidad regulativa de las leyes químicas es más cercana a la concepción kantiana de esas leyes que la lectura de McNulty.
\end{abstract}

Palabras Clave: Kant, ciencia en un sentido impropio, química, leyes empíricas, necesidad.

Abstract: The aim of this paper is to criticize an article by Michael B. McNulty published in 2015, namely, "Rehabilitating the Regulative Use of Reason: Kant on

\footnotetext{
* Profesor en Filosofía, Licenciado en Filosofía y Doctor en Humanidades y Artes con Mención en Filosofía por la Universidad Nacional de Rosario (Argentina). Realizó estudios posdoctorales en la Universidad Johannes Gutenberg de Mainz (Alemania) y en la Universidad Católica de Leuven (Bélgica). Se desempeña actualmente como Investigador Asistente del CONICET (Argentina). Sus investigaciones se ocupan de diferentes aspectos de la filosofía teórica kantiana. Dirección electrónica: arias.martin@gmail.com
} 
Empirical and Chemical Laws". In this article, McNulty examines Kant's conception of the necessity of empirical laws belonging to what, in the Metaphysical Foundations of Natural Science, is called science in an "improper" sense. McNulty considers the only science in an improper sense mentioned by Kant, namely, chemistry. In his article, McNulty presents an original and interesting interpretation of that conception. According to this interpretation, certain ideas of reason, which the author calls "elements", are the ground of the necessity of chemical laws. We will make three criticisms of this interpretation. 1) McNulty's thesis according to which from the perspective of reason chemical laws are strictly necessary is cognitively irrelevant for us. 2) McNulty never mentions any of Kant's texts as clearly supporting his interpretation of the ground of the necessity of chemical laws. 3) Our interpretation of the regulative necessity of chemical laws is closer to Kant's conception of these laws than McNulty's reading.

Keywords: Kant, science in an improper sense, chemistry, empirical laws, necessity.

\section{Introducción}

En su artículo de 2015 "Rehabilitating the Regulative Use of Reason: Kant on Empirical and Chemical Laws", ${ }^{1}$ Michael Bennett McNulty estudia la concepción kantiana del fundamento de la necesidad de una clase de leyes empíricas. ${ }^{2}$ Las leyes de esta clase pertenecen a lo que, en el "Prólogo" a MAN,

\footnotetext{
${ }^{1}$ McNulty, Michael, "Rehabilitating the Regulative Use of Reason: Kant on Empirical and Chemical Laws" ["Rehabilitando el uso regulativo de la razón: Kant acerca de las leyes químicas y empíricas”], Studies in History and Philosophy of Science, 54, 2015, pp. 110. Las traducciones de pasajes de este trabajo y de otros trabajos de comentaristas son nuestras.

2 Empleamos las siguientes abreviaturas de las obras de Kant: EEKU = Erste Einleitung in die Kritik der Urteilskraft (Primera introducción a la Critica de la facultad de juzgar), $\mathrm{KrV}=$ Kritik der reinen Vernunft (Crítica de la razón pura), $\mathrm{KU}=$ Kritik der Urteilskraft (Crítica de la facultad de juzgar), Log = Logik Jäsche (Lógica Jäsche), MAN = Metaphysische Anfangsgründe der Naturwissenschaft (Primeros principios metafísicos de la ciencia de la naturaleza) y V$\mathrm{Ph} /$ Danziger $=$ Danziger Physik (Física Danzig; notas de lecciones de física dadas por Kant durante el semestre de verano de 1785). Damos las referencias a KrV según la paginación de la primera $(1781=\mathrm{A})$ y la segunda $(1787=\mathrm{B})$ ediciones originales. Todas las referencias a otros textos de Kant son dadas de acuerdo con el tomo y la paginación de la edición académica $(\mathrm{AA}=$ Akademie-Ausgabe $)$ de las obras de Kant (Kant's gesammelte
} 
Kant denomina ciencia natural en un sentido "impropio" del término. Esta concepción de la ciencia en un sentido impropio se aplica, cuando menos, a la química, y se opone a la concepción de la ciencia en un sentido "propio", esto es, la física matemática. Esta física es una ciencia natural en sentido propio porque posee una parte metafísica que legitima la aplicación adecuada de la matemática dentro de su ámbito. Esta parte metafísica es el fundamento de la parte empírica de esta disciplina. La química, en cambio, es una ciencia natural en sentido impropio porque carece de esa parte metafísica. Por tanto, la matemática no puede aplicarse adecuadamente en el ámbito de esta disciplina y, como veremos, esta última está justificada solamente de modo empírico. ${ }^{3}$ Cabe destacar, además, que durante el período de $\mathrm{KrV}(1781 / 1787)$ y MAN (1786), Kant adhiere a la química flogística tradicional desarrollada

Schriften, Berlin, Walter de Gruyter y predecesores, 1900ss.). Las traducciones utilizadas son las siguientes: para EEKU, la de Nuria Sánchez Madrid (Primera Introducción de la Crítica del Juicio, Madrid, Escolar y Mayo, 2011); para KrV, la de Mario Caimi (Crítica de la razón pura, México, Fondo de Cultura Económica, 2009); y para MAN, la de Carlos Másmela (Principios metafísicos de la ciencia de la naturaleza, Madrid, Alianza, 1989). En algunas ocasiones hemos modificado detalles de estas traducciones a fin de ofrecer versiones más fieles a los originales alemanes.

${ }^{3}$ Kant indica que las leyes empíricas pueden alcanzar necesidad en A159/B198. En MAN (AA 04: 468) se dice lo siguiente: las leyes de la experiencia de la química "no llevan consigo ninguna conciencia de su necesidad (no son apodícticamente ciertas)." Estimamos que Kant parece afirmar que las leyes de la química no son necesarias porque intenta contrastarlas con leyes a priori y apodícticamente ciertas, tales como las de la parte metafísica de la física matemática, parte que presentará unas páginas más adelante (AA 04: 469-470). Como veremos en la cuarta sección de este trabajo, aunque las leyes de la química no sean apodícticamente ciertas, ellas pueden obtener una cierta necesidad. Acerca de la diferencia entre la ciencia en sentido propio y en sentido impropio, cfr. MAN (AA 04: 468-471), Blomme, Henny, "Pourquoi la chimie ne peutelle aspirer au titre de science proprement dite ?”, en Grapotte, Lequan y Ruffing (eds.), Kant et les sciences, Paris, Vrin, 2011, pp. 159-168 y "Kant's Conception of Chemistry in the Danziger Physik", en Clewis (ed.), Reading Kant's Lectures, Berlin, De Gruyter, 2015, pp. 484-502; y McNulty, Michael, "Kant on Chemistry and the Application of Mathematics in Natural Science", Kantian Review, 19, 3, 2014, pp. 393-418. Véase la segunda sección de este trabajo. 
particularmente por Georg Stahl (1660-1734). El objetivo de este trabajo es realizar una exposición crítica del estudio de McNulty. ${ }^{5}$

El examen del artículo de McNulty está justificado porque en el mismo se realiza una importante contribución. El autor expone una lectura novedosa e interesante de la naturaleza necesaria de las leyes empíricas de la ciencia en sentido impropio y confronta esta lectura con dos tipos de interpretaciones anteriores sobre la naturaleza necesaria de las leyes empíricas en general.

Examinaremos críticamente las tesis de McNulty en el siguiente orden. En la segunda sección, caracterizaremos las dos clases de interpretaciones anteriores, distinguidas por McNulty, acerca de la naturaleza necesaria de las leyes empíricas en general. La clase "categorial" de interpretación sostiene que esta necesidad puede derivarse únicamente de la aplicación de las categorías a ciertos contenidos empíricos, mientras que la clase "sistemática" mantiene que la necesidad mencionada proviene de una actividad de sistematización que tiende incesantemente a la obtención de una totalidad de conocimiento. Al final de esta sección resumiremos las objeciones principales que hace McNulty a estas dos clases de interpretaciones. La exposición efectuada en esta sección nos permitirá comenzar a tratar el problema de la necesidad de las leyes de la ciencia en sentido impropio.

En la tercera sección, examinaremos la clase "ideacional" de interpretación propuesta por McNulty. Nuestro autor considera fundamentalmente la química flogística, porque la química es la única ciencia en sentido impropio mencionada por Kant y en el período de $\mathrm{KrV}$ y MAN, como vimos, Kant es un seguidor de la química de esa clase. Según esta interpretación ideacional, la necesidad de las leyes pertenecientes a la química

\footnotetext{
${ }^{4}$ Cfr. Friedman, Michael, Kant and the Exact Sciences, Cambridge, Harvard University Press, 1992, pp. 240-242 y 264-267; Carrier, Martin, "Kant's Theory of Matter and His Views on Chemistry", en Watkins (ed.), Kant and the Sciences, Oxford, Oxford University Press, 2001, pp. 205-230; y Blomme, Henny, "Pourquoi la chimie ne peut-elle aspirer au titre de science proprement dite?" y "Kant's Conception of Chemistry in the Danziger Physik". Según Friedman, Kant habría adoptado la química antiflogística de Lavoisier a más tardar en 1795. Cfr. Friedman, Michael, Kant and the Exact Sciences, p. 289.

${ }^{5}$ No conocemos trabajos que analicen el artículo de McNulty.
} 
flogística resulta del empleo de ciertas ideas de la razón teórica que McNulty denomina "elementos". Ejemplos de ellos son las ideas de la sal y el flogisto. Estos elementos posibilitan explicaciones causales y unificadas de diversos fenómenos químicos. Asimismo, los elementos, en tanto ideas de la razón, son a priori. Por tanto, las leyes de la química, en la medida en que en su formulación se emplean elementos, son estrictamente universales y necesarias. Sin embargo, estas leyes poseen esa necesidad y esa universalidad solamente desde la perspectiva de la razón. Dado que las ideas de la razón tales como los elementos son incognoscibles, la universalidad y la necesidad estrictas de las leyes formuladas con elementos, como se desprende de lo dicho por McNulty mismo, también son incognoscibles. ${ }^{6}$ Esta universalidad y esta necesidad son cognitivamente irrelevantes para nosotros, porque poseemos una sensibilidad que no nos permite adquirir conocimiento de ideas de la razón. Por otro lado, puede afirmarse que la argumentación de McNulty acerca del fundamento de la universalidad y la necesidad de las leyes químicas no posee base textual suficiente. $^{7}$

En la cuarta sección, expondremos concisamente nuestra interpretación del fundamento de la necesidad de las leyes de la química flogística. Tal interpretación puede entenderse como una variante de la clase de interpretación que McNulty llama "sistemática". Según nuestra lectura, sólo las leyes químicas pertenecientes al sistema total de las mismas serían estrictamente necesarias. Asimismo, las leyes químicas con amplio sustento empírico e

\footnotetext{
${ }^{6} \mathrm{~A}$ fin de simplificar, no exponemos en esta presentación introductoria la distinción que hace McNulty entre los principios ideacionales de la química flogística y las leyes que pueden deducirse de ellos. Un ejemplo de principio ideacional es "el flogisto es la causa de la calcinabilidad de los fenómenos", y un ejemplo de ley que puede derivarse de este principio es "el flogisto es la causa de la calcinabilidad del estaño". Véase la tercera sección de este trabajo.

${ }^{7}$ Cabe destacar que McNulty dedicó otros trabajos a la concepción kantiana de la química (cfr. "Kant on Chemistry and the Application of Mathematics in Natural Science"; "Chemistry in Kant's Opus Postumum", HOPOS: The Journal of the International Society for the History of Philosopby of Science, 6, 1, 2016, pp. 64-95; y "What is Chemistry, for Kant?", en Heidemann, -ed.-, Kant Yearbook 9: Pbilosophy of Science, Berlin, Walter de Gruyter, 2017, pp. 85-112). Aquí examinamos solamente el artículo de 2015 porque es el único que brinda una interpretación detallada de la necesidad de las leyes pertenecientes a la química (cfr. supra, n. 1).
} 
integradas en un sistema parcial existente de estas leyes serían potencialmente necesarias, dado que es probable suponer que estas leyes podrían integrarse en un futuro ideal en el sistema total de las mismas. Denominaremos "regulativa" a esta necesidad potencial. Notemos que esta necesidad regulativa puede ser conocida por nosotros. Finalizaremos esta sección con una consideración de las objeciones hechas por McNulty a la clase sistemática de interpretación.

\section{Las clases de interpretación categorial y sistemática}

McNulty distribuye en dos clases las interpretaciones precedentes del carácter necesario de las leyes empíricas en general y las describe precisamente. Según la interpretación categorial de Michael Friedman, las leyes empíricas necesarias sólo pueden originarse especificando progresivamente con contenido empírico los principios del entendimiento puro expuestos en $\mathrm{KrV} .{ }^{8}$ Estos principios expresan la aplicación de las distintas categorías a diferentes aspectos de los fenómenos. Ejemplos de tales principios son las analogías de la experiencia. 9 Friedman sostiene que, en MAN, los principios del entendimiento puro son aplicados a un concepto empírico de materia, y que de esta aplicación surgen los principios a priori de la metafísica particular de la física matemática (por ejemplo, las leyes de la mecánica). ${ }^{10}$ Estos principios metafísicos son necesarios y universales porque están vinculados con los principios del entendimiento puro y, consiguientemente, con las categorías, y transmiten esa necesidad y universalidad a otras leyes pertenecientes a la física matemática. Por ejemplo, la ley empírica de gravitación universal es establecida mediante la aplicación de los principios de MAN a las regularidades empíricas descubiertas por Kepler. La parte a priori y fundamental de la física matemática se origina entonces, en última instancia, en las categorías, y es el fundamento de las leyes empíricas necesarias de esta disciplina. ${ }^{11}$ Para Friedman, otras

\footnotetext{
${ }^{8}$ Cfr. A148/B187ss.

${ }^{9}$ Cfr. A176/B218ss.

${ }^{10}$ Cfr. MAN (AA 04: 536ss.).

${ }^{11}$ Esta parte a priori y fundamental constituye igualmente el conocimiento metafísico que justifica la aplicación adecuada de la matemática en el ámbito de la física. Véase la primera sección de este trabajo.
} 
ciencias naturales pueden contener leyes empíricas necesarias sólo en la medida en que estas últimas puedan fundarse en los principios de la metafísica particular de la física matemática expuesta en MAN y, mediante estos últimos, fundarse en los principios del entendimiento puro de $\mathrm{KrV} .{ }^{12}$

Las interpretaciones sistemáticas, en cambio, resaltan que la necesidad de las leyes empíricas entraña un proceso de sistematización de las mismas que tiende asintóticamente a la consecución de una totalidad de conocimiento. Esta temática es expuesta por Kant, principalmente, en el "Apéndice a la dialéctica trascendental" de $\mathrm{KrV}$ y en las dos introducciones a KU. Mientras que Friedman sostiene que la sistematización de las leyes empíricas puede otorgarles necesidad sólo en tanto que las conecta, en última instancia, con las categorías, para las interpretaciones sistemáticas es la sistematización misma la que puede conferir necesidad. El sentido en el que la sistematización es fuente de necesidad varía en los distintos autores que sostienen esta clase de

${ }^{12}$ McNulty, 2 y 9. Damos las referencias al artículo de McNulty ("Rehabilitating the Regulative Use of Reason: Kant on Empirical and Chemical Laws") mediante el apellido del autor y los números de página. Los demás datos del texto fueron ofrecidos en supra, n. 1. A fin de simplificar, omitimos las abreviaturas "p." y "cfr". 
interpretación. ${ }^{13}$ En este contexto, ${ }^{14}$ McNulty menciona a Gerd Buchdahl, ${ }^{15}$ Philip Kitcher, ${ }^{16}$ Fred Rush ${ }^{17}$ y Paul Guyer. ${ }^{18}$

Resumiremos a continuación las críticas principales que McNulty efectúa a estas dos clases de interpretaciones. En primer lugar, la interpretación categorial no puede explicar adecuadamente la necesidad de las leyes empíricas pertenecientes a la ciencia en sentido impropio, puesto que es válida únicamente para las leyes empíricas de la ciencia en sentido propio. ${ }^{19} \mathrm{Si}$ bien no podemos explicar aquí esta cuestión, señalamos que estamos de acuerdo con esta crítica. En segundo lugar, las interpretaciones sistemáticas carecen de base textual suficiente y no explican claramente en qué sentido la aplicación progresiva de ciertos procedimientos de sistematización puede conferir

${ }^{13}$ Estimamos que, según $\mathrm{KrV}$, el mencionado proceso de sistematización es regido por el uso regulativo de dos diferentes tríadas de ideas de la razón teórica, a saber, las ideas como principios (los de la homogeneidad, la especificación y la continuidad) y como entes ideales (los del alma, el mundo y Dios). La primera tríada de ideas es expuesta en la primera parte del "Apéndice a la dialéctica trascendental" de KrV (A642-668/B670696), y la segunda es presentada en la segunda parte de esta sección (A669-704/B697732). Hemos examinado estas dos tríadas de ideas y sus relaciones mutuas en Arias Albisu, Martín, "Acerca de la relación entre los dos tipos de esquemas de las ideas de la razón en la Crítica de la razón pura de Kant", Areté, 24, 1, 2012, pp. 7-24. Por motivos de espacio, no haremos referencia aquí a la doctrina de la facultad de juzgar reflexionante de KU. Cfr. infra, n. 47, n. 62 y n. 67.

${ }^{14}$ McNulty, 2-3.

${ }^{15}$ Cfr. "The Relation between 'Understanding' and 'Reason' in the Architectonic of Kant's Philosophy", Proceedings of the Aristotelian Society, 67, 1967, pp. 209-226 y "The Conception of Lawlikeness in Kant's Philosophy of Science", Synthese, 23, 1, 1971, pp. 24-46.

${ }^{16}$ Cfr. "Projecting the Order of Nature", en Butts (ed.), Kant's Philosophy of Physical Science, Dordrecht, D. Reidel, 1986, pp. 201-235 y "The Unity of Science and the Unity of Nature", en Parrini (ed.), Kant and Contemporary Epistemology, Dordrecht, Kluwer, 1994, pp. 253-272.

${ }^{17}$ Cfr. "Reason and Regulation in Kant", The Review of Metaphysics, 53, 4, 2000, pp. 837862.

${ }^{18}$ Cfr. "Kant's Conception of Empirical Law", Proceedings of the Aristotelian Society, Supplementary Volumes, 64, 1990, pp. 221-242 y "Kant on the Systematicity of Nature: Two Puzzles”, History of Philosophy Quarterly, 20, 3, 2003, pp. 277-295.

${ }^{19}$ McNulty, 2. 
necesidad a las leyes empíricas. ${ }^{20}$ Consideraremos estas dos últimas críticas al final de la cuarta sección de este trabajo.

\section{La interpretación ideacional de McNulty: presentación y crítica}

\subsection{La postulación de los elementos puros}

La contribución fundamental del artículo de McNulty consiste en la propuesta de una interpretación de la necesidad de las leyes empíricas. Como adelantamos, el autor se ocupa principalmente de las leyes empíricas pertenecientes a la única ciencia en sentido impropio que Kant menciona, esto es, la química. Asimismo, como durante el período de $\mathrm{KrV}$ y MAN, según se ha visto, Kant adhiere a la química flogística, McNulty considera la química de esta clase. La interpretación del comentarista acerca de la necesidad de las leyes de la química flogística es novedosa con respecto a las dos clases de interpretaciones de las leyes empíricas en general que se esbozaron en la sección anterior. Según esta interpretación alternativa, que McNulty denomina "ideacional", la necesidad de las leyes de la química mencionada proviene del empleo de ciertas ideas de la razón teórica. Siguiendo algunos textos kantianos, McNulty denomina "elementos" a estas ideas. ${ }^{21}$ En el "Apéndice a la dialéctica trascendental" de su $\mathrm{KrV}$, Kant introduce esas ideas con ejemplos que, efectivamente, pertenecen a la química flogística, tales como los de "tierras", "sales" y "seres combustibles" 22 (este último hace referencia al flogisto). ${ }^{23}$ Estos elementos se originan en la razón "en lo que respecta a la completa pureza". ${ }^{24}$ Además, tales elementos puros, según Kant, son "causas naturales". ${ }^{25}$ McNulty interpreta que estos elementos son hipostatizaciones de poderes causales, y sostiene que ellos son postulados en número reducido a fin

\footnotetext{
${ }^{20}$ McNulty, 2-3.

${ }^{21}$ McNulty, 5.

22 "[B]rennliche Wesen". Caimi traduce: "cuerpos combustibles" (Kant, Immanuel, Crítica de la razón pura, p. 583). McNulty traduce: "inflammable beings" (McNulty, 5).

23 A646/B674.

24 Ibid.

${ }^{25}$ Ibid.
} 
de explicar unificadamente las propiedades químicas de una gran variedad de fenómenos. ${ }^{26}$

A continuación, ilustraremos el procedimiento en el marco del cual, según McNulty, se postula uno de esos elementos. Supongamos que queremos explicar causalmente la calcinabilidad del plomo. A fin de explicar de esa manera esta propiedad, empleamos lo que Kant denomina "uso hipotético de la razón". ${ }^{27}$ Este uso consiste, a grandes rasgos, en la búsqueda de una regla universal para ciertos particulares que están dados. Se podría, por ejemplo, considerar inicialmente un juicio particular a fin de buscar un principio a partir del cual el juicio mencionado pudiera seguirse. En el caso que nos ocupa, el juicio particular es "el plomo puede ser calcinado". Este juicio es una regularidad empírica basada en la observación. Usando hipotéticamente la razón, buscamos un principio que sostenga que otra de las propiedades del plomo es la causa de la calcinabilidad de este metal. En este punto, podemos plantear como hipótesis que una propiedad cualquiera del plomo es la causa de su calcinabilidad. ${ }^{28}$

Podríamos plantear que el color del plomo es la causa de su calcinabilidad. Se formularía entonces el siguiente principio: "las substancias grises pueden ser calcinadas". La calcinabilidad del plomo puede deducirse a partir de este principio y mediante la premisa menor "el plomo es gris". A fin de establecer si el principio formulado es universal, el investigador debe poner a prueba experimentalmente los juicios particulares que se siguen de este principio. De esta manera, el investigador podría averiguar que las cenizas, que son grises, no son calcinables. Esta falsación del juicio particular "las cenizas pueden ser calcinadas" implica que el principio "las substancias grises pueden ser calcinadas" no es universal y que la propiedad de ser gris no es la causa de la calcinabilidad. ${ }^{29}$

\footnotetext{
${ }^{26}$ McNulty, 5 y 8. Más adelante citamos el pasaje en el que McNulty introduce el concepto de elemento. Cfr. infra, n. 31.

${ }^{27}$ Cfr. A646-647/B674-675.

28 McNulty, 4.

${ }^{29}$ Ibid. McNulty señala acertadamente que Wartenberg, basándose en una lectura semejante del uso hipotético de la razón, considera que Kant adhiere a un "modelo hipotético-deductivo de la práctica científica” (McNulty, 4, n. 20; cfr. Wartenberg,
} 
McNulty también considera la hipótesis según la cual el carácter metálico del plomo es la causa de su calcinabilidad. Esta hipótesis parece a primera vista más prometedora, porque puede descubrirse experimentalmente que todos los metales conocidos son calcinables. Sin embargo, también puede descubrirse de esa manera que hay substancias no metálicas que pueden ser calcinadas, como por el ejemplo la caliza. Aunque esta circunstancia no contradice el principio "los metales son calcinables", ella pone en evidencia que la cualidad de ser un metal no puede ser la causa de la calcinabilidad en general. McNulty indica que nuestra razón considera juicios que están dados a fin de procurar encontrar condiciones absolutas para los mismos (el autor refiere a Bxx y A322326/B379-383). En el presente contexto, "la razón será satisfecha solamente con el fundamento último de la calcinación: una propiedad poseída por esas substancias que pueden ser calcinadas, y solamente por ellas." 30

Como no es posible identificar en la experiencia "ninguna propiedad que unifique todos los fenómenos de calcinación”, la razón, “a fin de satisfacer su propia necesidad de una condición incondicionada, propone una idea, el flogisto, como portador de la propiedad de calcinabilidad". 31

Thomas, "Order through Reason. Kant's Transcendental Justification of Science", Kant Studien, 70, 4, 1979, pp. 409-424, aquí pp. 410-411).

${ }^{30}$ McNulty, 4.

${ }^{31}$ McNulty, 5. "Las ideas, para Kant, son conceptos que están más allá de la posibilidad de la experiencia, pero [MAA: que son] postulados por la razón para sistematizar y explicar completamente nuestros conocimientos (KrV, A310-20/B366-77). Mientras que las ideas trascendentales de la razón (el alma, el mundo como una totalidad y Dios) son derivadas directamente a partir de las formas de la razón (las formas silogísticas), hay otras ideas, cuyos objetos están más allá de la posibilidad de la experiencia y que son postuladas por la razón en el contexto científico. Por ejemplo, Kant considera el espacio absoluto newtoniano (MAN, 4:563) y las especies biológicas ( $\mathrm{KrV}$, A317s./B374s.) como ideas que estructuran sus respectivos dominios. El espacio absoluto, aunque está más allá de la posibilidad de la experiencia, hace comprensible la noción de movimiento real. Kant entiende el flogisto como un elemento (V$\mathrm{Ph} /$ Danziger, 29:161-3; Refl 45, 14:371-96) y los elementos, en general, como ideas de la razón. Llama 'meros conceptos fundamentales de la razón' (Refl 45, 14:375) a los elementos y afirma que, en virtud de su pureza, los elementos surgen a partir de la razón: 'en lo que concierne a su completa pureza, [ellos] tienen su origen sólo en la razón' (KrV, A645s./B673s.). Para Kant, los elementos son postulados como 
El flogisto es un elemento puro que sería la causa de la calcinabilidad de los fenómenos. La presencia de flogisto en los fenómenos explicaría la calcinabilidad de estos últimos. Además, "como el flogisto es solamente el portador de la propiedad de calcinación, no tiene otras propiedades que puedan servir de fundamentos adicionales de la calcinabilidad". ${ }^{32}$ Los elementos puros como el flogisto están más allá de la posibilidad de la experiencia porque son "hipostatizaciones de poderes causales incondicionados". No puede darse en la experiencia "una substancia que porte solamente una propiedad química única". 33

McNulty presenta a continuación un experimento realizado por Georg Stahl y mencionado por Kant en $\mathrm{KrV} .{ }^{34}$ Este experimento proveería sustento para el principio según el cual el flogisto es el portador de la inflamabilidad. De acuerdo con este principio, el mismo flogisto que explica todos los fenómenos de calcinación, explica también todos los fenómenos de combustión. Para nuestros fines, lo importante es subrayar que la postulación del flogisto permite explicar propiedades químicas de una gran diversidad de fenómenos. ${ }^{35}$

substancias absolutamente puras que constituyen colectivamente los fundamentos básicos de las conexiones causales en la química (V-Ph/Danziger, 29:161). En KrV (A646/B674), afirma que en la química reducimos el comportamiento de las substancias a las substancias puras, incluyendo los 'seres inflamables' -esto es, el flogisto- y la sal, que son respectivamente las condiciones incondicionadas de la calcinabilidad y la acidez". (Ibid.). Los pasajes de Kant citados por McNulty fueron traducidos a partir de las versiones inglesas ofrecidas por este comentarista. "Refl" hace referencia a las notas privadas de Kant conocidas como Reflexiones. El número de tomo dado por McNulty en las referencias es el de la edición académica de las obras de Kant. Cfr. supra, n. 2.

32 McNulty, 5.

${ }^{33}$ McNulty, 5, n. 25. Puede afirmarse, aunque McNulty no lo diga explícitamente, que los fenómenos están siempre compuestos por mezclas de elementos puros. Cada uno de estos elementos portaría una propiedad química diferente y no podría ser experimentado aisladamente.

${ }^{34}$ Cfr. Bxii-xiii.

${ }^{35}$ McNulty, 5-6. Presentaremos sucintamente el experimento de Stahl. En primer lugar, Stahl calcinó plomo para obtener óxido de plomo. En la época de Stahl, este óxido era conocido como cal o ceniza de plomo. Stahl pensaba que esta calcinación tenía lugar mediante la liberación del flogisto contenido en el plomo. A continuación, Stahl quemó 
3.2. La necesidad racional de las leyes de la quimica y el problema de la incognoscibilidad de la misma

McNulty señala correctamente que, de acuerdo con Kant, las ideas de la razón son a priori (el autor remite a A320/B377). Los principios que consisten en la afirmación de la propiedad característica de una idea, tales como el principio del flogisto, a saber, "el flogisto es el portador de la inflamabilidad", son también a priori. Los principios a priori, para Kant, son estrictamente universales y necesarios (McNulty refiere a B4). Por tanto, los principios ideacionales, como el del flogisto, son también estrictamente universales y necesarios. ${ }^{36}$ Para McNulty, estos principios ideacionales de la química transmiten esta universalidad y esta necesidad estrictas a los juicios que pueden deducirse de ellos. Como se ha indicado, juicios como "el estaño es calcinable" son originalmente hipótesis basadas en observaciones. Sin embargo, la introducción del principio ideacional según el cual el flogisto es el portador de la inflamabilidad hace posible la deducción de ese juicio a partir del principio mencionado. Como los metales contienen flogisto y el estaño es una clase de metal, "cualquier muestra particular de estaño tiene que ser necesariamente

carbón vegetal en presencia del óxido de plomo y descubrió que este último se transformó en un trozo de plomo con sus características distintivas. Este proceso se explicaría por la liberación del flogisto contenido en el carbón vegetal y su absorción por parte del óxido de plomo. Este experimento brindaría sustento, como indica McNulty, al principio según el cual el flogisto es el elemento portador de la inflamabilidad, esto es, tanto los procesos de calcinación como los de combustión podrían explicarse por el mismo mecanismo de liberación de flogisto. Asimismo, el flogisto sería el mismo en los tres reinos de la naturaleza (por ejemplo, en el mineral plomo- y en el vegetal -carbón-). Acerca de este experimento, cfr. Stahl, Georg, Zufällige Gedancken und nützliche Bedencken über den Streit von dem so genannten Sulphure, Halle, Wäysenhaus, 1718, pp. 119-120; Partington, James, A History of Chemistry, London, McMillan, 1961, 2do vol, pp. 669-671; Carrier, Martin, "Kant's Theory of Matter and His Views on Chemistry", pp. 217-218 y McNulty (5-6). Acerca de este experimento y la probabilidad del principio que aparentemente sustenta, cfr. infra, n. 52.

${ }^{36}$ McNulty, 6. 
calcinable". ${ }^{37}$ McNulty mantiene que la necesidad que alcanzan juicios de nivel inferior como "el estaño es calcinable" es la necesidad de las leyes causales. El hecho de que estas leyes sean necesarias significa que las conexiones causales que ellas gobiernan son necesarias. Estas conexiones causales, a su vez, son necesarias en el sentido de que una determinación de un objeto denominada "efecto" se sigue indefectiblemente cada vez que se da otra determinación de un objeto denominada "causa". En el caso de las conexiones causales que nos ocupan, esto es, las pertenecientes a la química flogística, la necesidad mencionada se origina en el carácter ideal del elemento que, en última instancia, oficia de causa de ciertas determinaciones químicas. Por ejemplo, como hemos visto, el flogisto es la causa última de la inflamabilidad de los fenómenos. " $[\mathrm{L}] \mathrm{a}$ idealidad del flogisto hace necesarias [...] determinaciones de inflamabilidad de substancias orgánicas e inorgánicas, haciendo legales esas conexiones causales". 38

En la cuarta sección de su artículo, McNulty ofrece precisiones sobre su interpretación ideacional de la necesidad de las leyes químicas en general. El autor considera que los principios ideacionales no pueden ser conocidos en sentido estricto porque los elementos puros están más allá de la posibilidad de la experiencia. Sin embargo, puede creerse en esos principios. "Uno cree cuando asiente a un juicio con fundamentos subjetivos suficientes pero fundamentos objetivos insuficientes, mientras que uno conoce cuando asiente con

${ }^{37}$ Ibid. McNulty indica que las leyes de la química son o bien los mencionados principios ideacionales, o bien las regularidades empíricas de nivel inferior a las que ellos confieren necesidad (McNulty, 7). Por otro lado, dado que el flogisto es la causa de las diferentes inflamabilidades fenoménicas, y que del principio ideacional del flogisto pueden deducirse diversos juicios acerca de las diferentes inflamabilidades de los fenómenos, McNulty sostiene que los elementos puros son el fundamento lógico y causal-explicativo de toda la química (McNulty, 4 y 7). Señalemos asimismo que, en el marco de la exposición de la lectura de McNulty, expresiones como "leyes químicas en general" harán referencia conjuntamente a los principios ideacionales y a las leyes que pueden derivarse de ellos. En cambio, expresiones como "leyes (o juicios) de nivel inferior" se referirán solamente a estas últimas leyes.

${ }^{38}$ McNulty, 6. Cfr. infra, esp. n. 47. 
fundamentos subjetivos y objetivos suficientes". ${ }^{39}$ A continuación, McNulty introduce el concepto de "creencias teóricas". ${ }^{40}$ Estas creencias se sustentan en un tipo determinado de fundamentos subjetivos. De hecho, una creencia teórica "es hipotéticamente necesaria para la consecución de algún fin teórico". ${ }^{41}$ Según el autor, el principio ideacional del flogisto "hace posibles las leyes químicas de combustión y calcinabilidad, y entonces la creencia en este principio es así garantizada para la consecución de los fines de la química". ${ }^{42}$

El hecho de que los principios ideacionales no puedan ser conocidos tiene consecuencias para el estatus de los juicios o leyes que pueden derivarse de ellos. En efecto, esos principios no pueden ser los fundamentos epistémicos objetivos para esos juicios o leyes. Dice McNulty: "en el contexto de la justificación epistémica, la garantía (fundamentos objetivos) de los juicios de la química es siempre experiencial". ${ }^{43} \mathrm{El}$ autor explica que, aunque desde la perspectiva de la razón los juicios o leyes de nivel inferior se derivan necesariamente de los principios ideacionales que postulan elementos puros, como estos elementos son incognoscibles, la garantía epistémica de esos juicios o leyes es siempre "empirica o experimental". ${ }^{4}$ A diferencia, entonces, de las leyes fundamentales de la física matemática, que son apodícticamente ciertas, las leyes de la química derivadas de los principios ideacionales son "epistémicamente contingentes". 45

\subsection{Crítica de la doctrina de la necesidad racional de las leyes de la química}

\footnotetext{
${ }^{39}$ McNulty, 7. Cfr. A822/B850ss. En ese contexto, McNulty afirma que "creencia" (belief; Glaube) y "conocimiento" (knowledge; Wissen) son para Kant términos técnicos (McNulty, 7).

40 "[T] heoretical beliefs". El autor sigue a Andrew Chignell (cfr. "Belief in Kant", The Philosophical Review, 116, 3, 2007, pp. 323-360). No podemos presentar aquí el contenido completo del trabajo de Chignell.

${ }^{41}$ McNulty, 7.

${ }^{42}$ McNulty, 8.

43 McNulty, 8-9.

${ }^{44}$ McNulty, 9.

${ }^{45}$ Ibid. Cfr. infra, n. 50.
} 
El esfuerzo realizado por McNulty para sostener, sólida y persuasivamente, la doctrina según la cual los principios ideacionales son el fundamento a priori de la química parece mostrarse inútil cuando el autor presenta su tesis según la cual estos principios son meras creencias teóricas. Esta tesis no entraña solamente la incognoscibilidad de la universalidad y la necesidad de esos principios, sino también la incognoscibilidad de la universalidad y la necesidad de las leyes que pueden deducirse de ellos.

Recordemos que anteriormente se indicó que, para McNulty, las leyes que pueden seguirse de los principios ideacionales de la química adquieren la universalidad y la necesidad de estos últimos. El autor asevera: "la derivación de un juicio a partir del principio flogístico excluye la posibilidad de excepciones, haciendo al juicio estrictamente universal: contener flogisto es solamente ser inflamable. Todos esos juicios particulares -que el carbón es combustible, que el plomo es calcinable- son necesarios en virtud de ser consecuencias de la ley flogística fundamental". ${ }^{46}$ Resulta difícil conciliar este pasaje con las tesis de que estos principios ideacionales son incognoscibles y de que los juicios o leyes de nivel inferior están justificados epistémicamente sólo de manera experiencial. Podría señalarse, adoptando el punto de vista de la razón, que el flogisto no es más que una hipostatización del poder causal de la inflamabilidad y que, por tanto, todos los fenómenos que contienen flogisto son necesariamente inflamables. Ahora bien, en este caso lo que debe justificarse experimentalmente es que todos los fenómenos inflamables poseen flogisto y que no poseen otra propiedad diferente que también pueda ser causa de su inflamabilidad. ${ }^{47}$

\footnotetext{
${ }^{46}$ McNulty, 6.

${ }^{47}$ En EEKU, Kant dice: “¿Podía empero Linneo esperar esbozar un sistema de la naturaleza si hubiera tenido que preocuparse de que, si encontraba una piedra que llamaba granito, ésta pudiese ser diferente según su constitución interna de toda otra [MAA: piedra] que sin embargo tuviese justamente la misma apariencia, y entonces él pudiese esperar encontrar siempre sólo cosas singulares, en cierto modo aisladas para el entendimiento, pero nunca una clase de las mismas que pudiese ser llevada bajo conceptos de género y especie?" (AA 20: 215-216). Interpretamos este pasaje en el sentido de que, a fin de formar y emplear un concepto empírico, es necesario presuponer homogeneidad de constitución interna en los casos particulares del mismo (cfr. la presentación del "principio de homogeneidad" en el "Apéndice a la dialéctica
} 
Los principios ideacionales son incognoscibles y las leyes de nivel inferior que pueden derivarse de ellos están, entonces, epistémicamente justificadas sólo de manera experiencial. ${ }^{48}$ Por tanto, es posible que se encuentren casos particulares que contradigan a estas leyes de nivel inferior. Efectivamente, la experiencia no puede justificar ni la necesidad ni la universalidad que poseerían las leyes mencionadas desde la perspectiva de la razón. Como la universalidad y la necesidad estrictas son inseparables ${ }^{49}$ nos bastará con señalar que no puede confirmarse la universalidad de estas leyes. Puede afirmarse que, para Kant, esta universalidad es inverificable porque no puede demostrarse que todas las consecuencias posibles de una hipótesis empírica se correspondan con los fenómenos, dado que estas consecuencias son ilimitadas. ${ }^{50}$ Notemos, sin embargo, que las hipótesis empíricas pueden

trascendental" de KrV; A651-654/B679-682). Podría afirmarse que, para Kant, la formación y el empleo del concepto de metal entrañan, como condición de su posibilidad, la presuposición de que todos los metales poseen alguna clase de homogeneidad en lo que hace a su constitución interna. Sin embargo, el hecho de que pretendamos haber mostrado que ciertos metales poseen flogisto no implica necesariamente que todos los metales posibles lo contengan. Kant da a entender en más de una ocasión y muy claramente que, aunque tengamos que presuponer a priori la unidad sistemática del conocimiento como perteneciente a la naturaleza misma, no podemos determinar a priori el grado de esta unidad sistemática que encontraremos en la naturaleza en un momento dado (cfr. A653/B681, A654/B682, A661/B689, A663/B691, A664/B692, A665/B693, A668/B696 y A669/B697). Cfr. supra, n. 13 e infra, n. 62 y n. 67.

${ }^{48}$ McNulty, 8-9.

${ }^{49}$ Cfr. B3-4.

${ }^{50}$ Para McNulty, no sólo los principios ideacionales, sino también las leyes de nivel inferior son formuladas mediante elementos. Un ejemplo de estas hipótesis de leyes de nivel inferior es "el flogisto es el fundamento de la calcinabilidad del plomo". Sin embargo, hemos visto que, según McNulty, estas hipótesis son de naturaleza empírica en lo que hace a su justificación epistémica. Intentaremos explicar con más detalle este punto. Aunque, por ejemplo, el flogisto sea una idea, el referente de esta idea es pensado como contenido en ciertos fenómenos, tales como, según el ejemplo dado, las muestras de plomo. El investigador no puede encontrar el flogisto en su pureza en esos fenómenos, pero supone que el mismo se encuentra en ellos, si bien siempre mezclado con otras materias u otros elementos que poseen propiedades químicas diferentes (notemos que McNulty sostiene que los elementos están más allá de la posibilidad de 
volverse más probables en virtud de la puesta a prueba empírica exitosa de las mismas. ${ }^{51}$

No pensamos que McNulty se equivoque cuando afirma que la justificación epistémica de las leyes químicas susceptibles de derivarse de principios ideacionales es experiencial. Por el contrario, consideramos que es la tesis según la cual estas leyes son, desde el punto de vista de la razón, universales y necesarias, la que es cuestionable. Más precisamente, esta tesis es cognitivamente irrelevante para nosotros, porque tenemos una intuición meramente sensible. Dado que nuestra sensibilidad no nos permite conocer ideas de la razón como los elementos, sino tan sólo objetos empíricos, la universalidad y la necesidad que estos elementos transmitirían a las leyes químicas de nivel inferior no poseen ninguna incidencia en nuestro conocimiento de los fenómenos químicos. Es asimismo evidente que esta incognoscibilidad de los elementos también entraña la incognoscibilidad de la universalidad y la necesidad de los principios ideacionales que afirman las propiedades características de estos elementos. De hecho, como vimos en la

la experiencia no meramente porque sean substancias puras no adulteradas, sino principalmente porque poseen una propiedad química única. Cfr. la primera parte de esta sección, esp. n. 33 y McNulty, 5, n. 25). Dado que se supone que los elementos están contenidos en los fenómenos, las hipótesis empíricas de leyes de nivel inferior formuladas con estos elementos deben ser confrontadas con lo dado en la experiencia. Hemos examinado la naturaleza de las hipótesis empíricas en otro trabajo. Cfr. Arias Albisu, Martín, "Las prescripciones metodológicas de la función regulativa de la razón teórica en la 'Crítica de la razón pura' de Kant”, Kant e-Prints, Serie 2, 10, 1, 2015, pp. 64-93. Acerca de la posible confrontación con la experiencia en el caso de los principios ideacionales, cfr. infra, n. 52.

${ }^{51}$ Para Kant, cuando se encuentran, sea por experimentación o por observación rigurosa, casos particulares que están de acuerdo con una hipótesis empírica, ella se torna más probable. Aunque estas hipótesis, consiguientemente, no puedan ser conocidas como universales, la puesta a prueba empírica exitosa de las mismas hace que ellas puedan acercarse progresivamente a la universalidad. Acerca de la probabilidad de las hipótesis y su carácter no universal, cfr. A646-647/B674-675, A790-791/B818-819 y Log (AA 09: 84-86). Parecería que, según Log, sólo en el ámbito de la matemática puede la probabilidad expresarse numéricamente (cfr. Log, AA 09: 82). Acerca de la tesis de McNulty según la cual no puede atribuirse probabilidad a los principios ideacionales, cfr. infra, n. 52. 
segunda parte de esta sección, para McNulty no podemos tener conocimiento de los principios ideacionales, sino tan sólo creencias teóricas acerca de los mismos. ${ }^{52}$ Nuestra crítica principal a la interpretación de McNulty es, entonces, que su tesis acerca de la universalidad y la necesidad de las leyes químicas en general es cognitivamente irrelevante para nosotros.

Por otro lado, puede efectuarse igualmente una crítica interna a la concepción de McNulty. Como vimos en la segunda parte de esta sección, el autor basa su argumentación acerca de la universalidad y la necesidad de las leyes químicas en general en el carácter a priori de las ideas de la razón en general. Dado que los elementos son ideas de la razón, la argumentación del autor no parecería ser ilegítima. Sin embargo, la presentación de un texto de Kant en el que se conectasen los elementos con la universalidad y/o la necesidad de las leyes químicas o, en general, empíricas, habría provisto mucho más sustento a la interpretación de nuestro comentarista. ${ }^{53}$

${ }^{52}$ Cuando introduce la temática de las creencias teóricas, nuestro comentarista dice lo siguiente: "[c]omo el flogisto no es un objeto posible de la experiencia, no se pueden tener fundamentos objetivos suficientes para su existencia; esto es, ninguna experiencia hará la existencia del flogisto más o menos probable" (McNulty, 8). No estamos de acuerdo con esta afirmación de McNulty. El hecho de que no podamos conocer en sentido estricto los elementos puros ni, consiguientemente, los principios ideacionales, no implica que no podamos acercarnos asintóticamente a este conocimiento mediante la puesta a prueba empírica exitosa de las leyes de nivel inferior que pueden deducirse de estos principios. Puede afirmarse que la realización del experimento de Stahl mencionado anteriormente (cfr. supra, n. 35) provee sustento empírico a la siguiente ley de nivel inferior: "el flogisto es la causa de la calcinabilidad del plomo y de la combustibilidad del carbón vegetal". Este experimento torna más probable esta ley de nivel inferior, y de esta manera se torna también más probable el principio ideacional del cual puede derivarse esa ley de nivel inferior, a saber, el principio ideacional del flogisto. Según este principio, el flogisto es la causa de la calcinabilidad y la combustibilidad en general. No desarrollaremos esta objeción a la interpretación de McNulty, porque nos interesa principalmente mostrar que la universalidad y la necesidad que, según este comentarista, poseerían las leyes químicas en general no pueden ser conocidas por nosotros.

${ }^{53}$ Cabe destacar que McNulty cita un pasaje de V-Ph/Danziger (AA 29: 161) a fin de mostrar que también elementos diferentes del flogisto pueden explicar propiedades químicas (McNulty, 7). En ese pasaje, antes de que se haga mención de los elementos, 
Antes de concluir esta sección, quisiéramos señalar que estamos de acuerdo con una importante tesis de McNulty que es independiente de su concepción de la universalidad y necesidad de las leyes químicas. Indica correctamente el autor que el empleo de elementos puros tiene como meta ideal la explicación de todos los fenómenos químicos mediante unos pocos de esos elementos. ${ }^{54}$ Para Kant, "el fin principal y característico de la ciencia es la máxima unificación de la naturaleza". 55

\section{Una interpretación sistemática alternativa: la doctrina de la necesidad regulativa de las leyes de la química}

En otros lugares hemos delineado una interpretación propia de la necesidad de las leyes de la química flogística. ${ }^{56}$ Según esta interpretación, estas leyes pueden alcanzar en el marco de nuestra experiencia finita una necesidad que denominamos "regulativa". Esta concepción es compatible con la posibilidad de que en la experiencia se encuentren excepciones a las leyes regulativamente necesarias. Asimismo, la necesidad regulativa mencionada puede ser conocida por seres con una intuición meramente sensible como nosotros. Presentaremos brevemente una versión modificada de esta interpretación. Esta lectura puede entenderse como un caso particular de la clase de interpretación que McNulty denomina "sistemática". 57

se alude, según la traducción de McNulty, a una "necesidad” que estaría vinculada con una "división" efectuada por el entendimiento o la razón y que antecede a la experiencia. Esta división sería la que permite distinguir entre los diferentes elementos. De todas maneras, la necesidad mencionada no es referida a leyes empíricas.

54 McNulty, 8.

55 Ibid.

${ }^{56}$ Cfr. Arias Albisu, Martín, "Las prescripciones metodológicas de la función regulativa de la razón teórica en la 'Crítica de la razón pura' de Kant”, pp. 82-83, n. 43; y “Acerca del alcance objetivo de las prescripciones metodológicas de la función regulativa de la razón teórica en la Crítica de la razón pura de Kant”, Revista de Estudios Kantianos, 2, 2, 2017, pp. 149-167, aquí p. 159, n. 47.

${ }^{57}$ No disponemos de espacio para establecer si esta interpretación es también aplicable a leyes empíricas de ciencias diferentes de la química. 
Puede afirmarse que, para Kant, únicamente las leyes empíricas que sean parte del sistema completo de estas leyes podrían ser entendidas como estrictamente necesarias. "Esta idea [MAA: de la forma de un todo del conocimiento] postula, según eso, una unidad completa del conocimiento que es propio del entendimiento, en virtud de la cual ese [conocimiento] no se constituye como un mero agregado contingente, sino que llega a ser un sistema interconectado según leyes necesarias." 58 Asimismo, consideramos que la explicación, ofrecida por Fred Rush, del motivo por el cual las leyes empíricas pertenecientes al sistema completo de las mismas serían rigurosamente necesarias es plausible. Según Rush, mientras más se extiendan las leyes de un sistema en las direcciones de la generalidad y la especificidad, y mientras más leyes interconectadas estén incluidas en este sistema, más se aproxima este último al ideal de completitud. Aunque ningún sistema de leyes empíricas pueda adquirir en nuestra experiencia finita una forma completa y ser entonces verdaderamente necesario, cuando un sistema es progresivamente ampliado "tiende a descartar caracterizaciones empíricas competidoras de la naturaleza. $\mathrm{Y}$, en la medida en que hace eso, el sistema y sus leyes parecen exclusivos y $a$ fortiori necesarios." 59

El mencionado sistema completo sería, entonces, verdaderamente necesario en virtud de su exclusividad. Tal sistema no puede ser dado en nuestra experiencia finita, pero funciona como una meta ideal que orienta necesariamente todas las acciones empíricas de nuestro entendimiento. Estimamos que una ley química con un sustento empírico amplio e integrada

58 A645/B673. Una tesis similar parece encontrarse en KU (AA 05: 179-180). Asimismo, Kant vincula sistematicidad y verdad de "reglas" del entendimiento en A647/B675. Queda claro en los contextos de estas tres referencias que Kant no se refiere a los principios del entendimiento puro, sino fundamentalmente a las leyes empíricas de esta facultad. No tenemos espacio para referirnos a los contextos de estas referencias ni para estudiar la temática de sistemas de elementos o de conceptos empíricos. Tratamos con más detalle esas referencias y esos sistemas en Arias Albisu, Martín, "Las prescripciones metodológicas de la función regulativa de la razón teórica en la 'Crítica de la razón pura' de Kant”. Cfr. infra, n. 60.

${ }^{59}$ Rush, Fred, "Reason and Regulation in Kant", p. 847. Tesis similares pueden encontrarse en Kitcher, Philip, "The Unity of Science and the Unity of Nature", pp. 267-268. 
en un sistema parcial existente de leyes de esta clase puede considerarse como potencialmente necesaria, porque en este caso es probable suponer que esa ley podría llegar a formar parte del sistema completo de esas leyes. ${ }^{60}$ Mientras más sustento empírico posean esa ley y el sistema parcial existente en el que ella estaría integrada, y mientras más extenso y unificado sea este sistema parcial existente, más probabilidad tiene la suposición de que en un futuro ideal esa ley sería integrada en el sistema completo de las leyes de esa clase. Sin embargo, la ley en cuestión podría ser falsada en algún momento futuro real. Por tanto, las leyes de la química no pueden adquirir en nuestra experiencia finita una necesidad estricta, sino solamente, como indicamos, potencial. Esta necesidad potencial puede ser alcanzada, en principio, por todas las leyes de la química flogística, porque, a diferencia de McNulty, estimamos que también las leyes

${ }^{60}$ Con expresiones como "ley química con sustento empírico amplio" nos referimos a las leyes de la química que no fueron falsadas y que fueron puestas a prueba empíricamente con éxito en numerosas oportunidades. Cfr. supra, n. 51. Dado que en el "Apéndice a la dialéctica trascendental" de $\mathrm{KrV}$ se presentan ejemplos de sistematización en el ámbito de la química flogística (cfr. A652-653/B680-681 y A657/B685), es claro que esta disciplina tiene una forma sistemática. Cfr. también MAN (AA 04: 468-471). Por último, podría objetarse que esta concepción de necesidad no es aplicable a las leyes de la química flogística, porque estas leyes emplean elementos producidos por la razón, y en el pasaje citado en el que se introduce el concepto de necesidad vinculado con una sistematicidad completa, Kant hace referencia a leyes y conocimientos del entendimiento. Cfr. A645/B673 y supra, n. 58. No disponemos de espacio para responder detalladamente a esta objeción. Sin embargo, puede señalarse, en primer lugar, que, aunque los elementos sean ideas de la razón, ellos son pensados como contenidos en los fenómenos. Emplear elementos entraña hacer una referencia cognitiva unificada a una multiplicidad de fenómenos. Las leyes formuladas con estos elementos deben, por tanto, confrontarse con los fenómenos. Cfr. supra, n. 50, n. 51 y n. 52. En segundo lugar, tal vez las leyes de la química flogística pertenezcan al entendimiento en el sentido de que, al igual que las otras leyes empíricas, ellas pueden concebirse como particularizaciones de los principios del entendimiento puro. Cfr. A127-128, B165, A159/B198 y A216/B263. En la segunda sección de este trabajo, hemos visto que, según Friedman, las leyes empíricas necesarias de la física matemática resultan de la aplicación de los principios del entendimiento puro a ciertos contenidos empíricos. Aunque esta derivación no sea posible en el caso de leyes de una ciencia en sentido impropio como la química, estimamos que estas leyes son igualmente particularizaciones de esos principios. 
que este comentarista denomina "principios ideacionales" pueden adquirir sustento empírico objetivo. ${ }^{61}$ Por otro lado, proponemos denominar "regulativa" a esta necesidad potencial porque la tarea de perseguir asintóticamente la unidad sistemática total de los conocimientos del entendimiento es impuesta por la función regulativa de la razón teórica. ${ }^{62}$

Notemos que parece haber un sentido en el cual el empleo de los elementos de McNulty es una condición de la posesión de necesidad por parte de las leyes de la química. Como mostramos, las leyes formuladas mediante elementos permiten explicaciones unificadas de diferentes determinaciones químicas de una gran variedad de fenómenos. Por tanto, estos elementos podrían entenderse como condiciones de posibilidad de la formación de sistemas con unas pocas leyes químicas fundamentales en su cúspide. Un sistema de esta clase es el de la química flogística de la época de Kant. ${ }^{33}$ Sin embargo, incluso aunque esta tesis fuese correcta, la necesidad de las leyes de la química provendría en sentido estricto de la sistematización que los elementos harían posible, y no de los elementos mismos. Como se ha visto, la necesidad estricta de una ley química se define por la integración de esta última en el sistema ideal completo de leyes químicas, y la necesidad regulativa de una ley química se define por la probabilidad de la suposición según la cual esta ley sería integrada en un futuro ideal en ese sistema completo. Por consiguiente, aunque el uso de elementos fuese una condición de posibilidad de la construcción de sistemas de leyes químicas, la necesidad de las mismas se

${ }^{61}$ Recordemos que, según McNulty, los principios ideacionales son meras creencias teóricas y no poseen fundamentos objetivos. Cfr. la tercera sección de este trabajo. Acerca de la probabilidad y el sustento empírico objetivo de las leyes de nivel inferior y los principios ideacionales, cfr. supra, n. 51 y n. 52. Por otro lado, señalemos que en esta sección distinguiremos entre principios ideacionales y leyes de nivel inferior únicamente cuando hagamos referencia a la interpretación ideacional de McNulty.

62 Cfr. A644-645/B671-672. Hemos mencionado las dos tríadas de ideas sistematizadoras de la razón teórica en la nota 13 del presente trabajo. Cfr. también supra, n. 47 e infra, n. 67. No disponemos de espacio para introducir la doctrina de KU sobre la sistematización. Acerca de los elementos de McNulty como posibles condiciones de la sistematización, cfr. infra.

${ }^{63}$ Acerca de esta unificación, véase el experimento de Stahl presentado más arriba (cfr. supra, n. 35). Cfr. también McNulty, 8. 
definiría, a grandes rasgos, por el concepto de la integración de ellas en el sistema ideal completo. ${ }^{64}$

Podría objetarse, en primer lugar, que esta concepción de la necesidad regulativa de las leyes de la química flogística consiste meramente en la tesis de que algunas de estas leyes podrian ser estrictamente necesarias si, en un futuro ideal, fuesen integradas en el sistema total de las mismas. Debe notarse que, incluso si se acepta esta objeción, la concepción mencionada permitiría efectuar una distinción provechosa entre dos grupos de leyes químicas. De un lado, tendríamos las leyes químicas sin sustento empírico o con sustento empírico escaso, y/o sin posibilidad de integrarse en un sistema existente de estas leyes. Del otro lado, tendríamos las leyes químicas con sustento empírico amplio e integradas en un sistema existente. Tan sólo estas últimas pueden considerarse como regulativamente necesarias, porque la suposición de que ellas formarán parte del sistema ideal total está en este caso más justificada.

Parecería posible objetar, en segundo lugar, que en nuestra interpretación se encuentra una tensión semejante a la que presenta la lectura de McNulty. Esta última lectura contiene una tensión entre, por un lado, la necesidad que poseerían las leyes químicas de nivel inferior desde el punto de vista de la razón y gracias al empleo de elementos y, por el otro, la contingencia de las mismas en lo que haría a su justificación epistémica. Estas leyes serían epistémicamente contingentes porque los elementos son incognoscibles. A primera vista, podría afirmarse que nuestra interpretación contiene una tensión similar entre la incognoscible necesidad estricta de las leyes químicas y la cognoscible necesidad regulativa de las mismas. Sin embargo, estimamos que entre estas dos clases de necesidad no hay tensión, sino más bien conexión. El concepto de necesidad estricta de una ley química, que consiste en la integración de esta ley en el sistema ideal completo, permite definir el concepto de necesidad regulativa, porque ésta consiste, a grandes rasgos, en la probabilidad de la suposición de que una ley química sería integrada en un futuro ideal en ese sistema completo. Esta probabilidad puede ser conocida por nosotros, porque depende del sustento empírico amplio de la ley en cuestión y de la integración de ella en un sistema parcial existente.

${ }^{64}$ Cfr. infra, n. 67. Acerca de la escasa base textual de la interpretación de McNulty, cfr. supra, n. 53 y el párrafo en el que está incluida la llamada a esta nota. 
Al final de la segunda sección de este trabajo señalamos que, para McNulty, las interpretaciones sistemáticas no poseen base textual suficiente y no explican en qué sentido la sistematización puede conferir necesidad. La primera de estas críticas parece aplicable a la lectura que acabamos de exponer. Es cierto que nuestra concepción tiene más sustento textual que la interpretación de McNulty. Sin embargo, aunque Kant conecte sistematicidad con necesidad de leyes empíricas, nuestra distinción entre una necesidad estricta y una necesidad regulativa no aparece explícitamente en los textos kantianos. ${ }^{65}$ Con respecto a la segunda crítica, notemos que nuestra lectura tiene el mérito de mostrar claramente en qué sentido la sistematización puede otorgar necesidad a las leyes empíricas de la química. ${ }^{66}$ Por otro lado, la necesidad regulativa de las leyes químicas, a diferencia de la necesidad que ellas poseerían según McNulty, puede ser conocida por seres dotados de una intuición sensible como nosotros. Por consiguiente, aunque Kant no haya presentado explícitamente nuestra concepción en su totalidad, ella permite explicar, en lo que respecta cuando menos a la química flogística, la tesis kantiana según la cual leyes causales empíricas pueden alcanzar cierta necesidad. ${ }^{67}$

${ }^{65}$ Cfr. supra, n. 53 y párrafo en el que está incluida la llamada a la nota, n. 58 y párrafo en el que está incluida la llamada a la nota, y n. 60.

${ }^{66}$ McNulty menciona concisamente la interpretación de Rush presentada más arriba, pero aun así afirma que "no es claro cómo los mecanismos de los sistematizadores [...] podrían hacer necesarios los juicios de una ciencia" (McNulty, 3).

${ }^{67} \mathrm{Cfr}$. supra, n. 57. McNulty también señala que las interpretaciones sistemáticas no son compatibles con la tesis kantiana según la cual "tiene que haber [MAA: fuentes o] fundamentos a priori de la necesidad de las leyes empíricas" (McNulty, 3. El comentarista remite a A159/B198 y KU, AA 05: 179-180). Nuestro autor sostiene asimismo que la afirmación de que "la idea a priori de sistematicidad, en sí misma, hace necesarias las leyes de ciencias no físicas" no es acertada, porque "ni la idea de sistematicidad ni sus principios están en una relación inferencial directa con leyes naturales" (McNulty, 3). Según nuestra interpretación, en pocas palabras, la integración de leyes químicas en sistemas es la fuente de la necesidad de estas leyes. Efectuaremos dos aclaraciones. En primer lugar, a diferencia de lo que parece sostener McNulty, no consideramos que en los textos kantianos mencionados por este comentarista se afirme inequívocamente que las leyes empíricas necesarias de ciencias no físicas deban poder inferirse a partir de la fuente de la necesidad de estas leyes. En segundo lugar, tal vez pueda afirmarse que las ideas de la razón teórica, cuya aplicación es necesaria para la 


\section{Conclusiones}

En este artículo, principalmente, se expuso y criticó la interpretación, propuesta por McNulty, del fundamento de la necesidad de las leyes empíricas pertenecientes, específicamente, a la ciencia en sentido impropio que es la química flogística. Según esta interpretación ideacional, esta necesidad resulta del empleo de ciertas ideas de la razón teórica que McNulty denomina "elementos". Sin embargo, como los elementos, en tanto ideas de la razón, no pueden ser conocidos, los principios ideacionales que postulan a estos elementos tampoco pueden ser conocidos. Por tanto, no podemos conocer ni la universalidad ni la necesidad de estos principios. Nuestro comentarista también examina las leyes de nivel inferior que pueden deducirse de esos principios ideacionales. En virtud del empleo de elementos y desde la perspectiva de la razón, estas leyes de nivel inferior son universales y necesarias. No obstante, como los elementos son incognoscibles, la universalidad y la necesidad mencionadas son igualmente incognoscibles. Efectivamente, la justificación epistémica de esas leyes de nivel inferior depende siempre de lo

formación de sistemas de leyes químicas, sean condiciones a priori (aunque no fuentes) de la necesidad en cuestión (cfr. supra, n. 13, n. 47 y n. 62. Como adelantamos, no presentamos el concepto de facultad de juzgar reflexionante de KU por motivos de espacio). Vimos que también la aplicación de los elementos podría ser necesaria para la formación de estos sistemas. En este caso, los elementos también serían condiciones de esa necesidad. El punto es que, como las ideas mencionadas, y posiblemente los elementos, son condiciones a priori de la construcción de sistemas de leyes químicas, y la necesidad de estas leyes consiste en su integración en sistemas, entonces estas ideas, y posiblemente los elementos, son también condiciones de esta necesidad. Por último, McNulty asevera: "[f]inalmente, las interpretaciones sistemáticas amenazan con hacer colapsar la distinción de Kant entre las ciencias -cuerpos sistemáticos de conocimientos- y las ciencias racionales -aquéllas con leyes causales-. Si la sistematicidad produce leyes genuinas que tienen necesidad, entonces toda ciencia, en virtud de ser sistemática, sería también una ciencia racional” (McNulty, 3). Kant introduce los conceptos de ciencia y ciencia racional a los que se refiere McNulty en MAN (AA 04: 467-468). No es necesario examinar aquí esta objeción de McNulty, porque nos interesa solamente la sistematicidad de una ciencia que posee de por sí leyes causales, a saber, la química flogística. 
dado en la experiencia. Por tanto, la doctrina de McNulty acerca del carácter universal y necesario de las leyes químicas en general no tiene ninguna relevancia cognitiva para nosotros, ya que nuestra sensibilidad no nos permite conocer ideas de la razón, sino solamente fenómenos. Y, aun si esta doctrina fuese relevante, podría objetarse que McNulty no la fundamenta específicamente en los elementos, sino en la índole a priori de las ideas de la razón en general.

Posiblemente nuestra concepción de la necesidad regulativa de las leyes químicas en general, que hemos presentado en este artículo, sea superior a la interpretación de McNulty. Esta necesidad regulativa puede ser conocida por nosotros, dado que depende del sustento empírico amplio de una ley química y de su integración en un sistema parcial existente de leyes de esta clase. Además, nuestra lectura es compatible con la posibilidad de que la experiencia ofrezca excepciones a las leyes químicas regulativamente necesarias y permite efectuar una distinción útil entre dos diferentes grupos de leyes de la química.

Recibido: 02/2018; aceptado: 05/2018 